\title{
L'influence du contexte sur les pratiques gestuelles du jeune enfant lors d'explications en « comment »
}

\author{
Mazur-Palandre, Audrey \& Lund, Kristine \\ Laboratoire ICAR (UMR5191) ; CNRS ; Université Lyon 2 ; ENS de Lyon \\ Audrey.Mazur_Palandre@ens-lyon.fr \& Kristine.Lund@ens-lyon.fr
}

\section{Introduction}

Le présent travail ${ }^{1}$ a pour but d'étudier l'activité langagière, plus précisément l'explication, de jeunes enfants (6 ans). Étudier l'activité langagière de l'enfant entend la prise en considération des choix linguistiques des enfants et également des gestes qu'ils produisent lors de la production d'un message (entre autres, Colletta, 2000, 2004, 2007, 2009 ; Colletta et Pellenq, 2005 ; Colletta, Pellenq et Guidetti, 2010 ; Colletta, Venouil, Kunene, Kaufmann, Simon, 2009 ; Cosnier, 1996, 2008 ; Fantazi, 2010 ; Fantazi et Colletta, 2010 ; Graziano, 2010 ; Kendon, 2004 ; McNeill, 1992, 2000). Pour ce papier, l'intérêt porte spécifiquement sur la production des gestes des enfants lors d'explication «en comment». La méthode est quasi-expérimentale dans la mesure où certains aspects du contexte de production sont manipulés. Ainsi, les enfants ont réalisé, en binôme, deux tâches imposées appelant des ressources cognitives différentes (une tâche numérique et une tâche de spatialité). L'expérimentation s'est déroulée en trois phases : (a) dans un premier temps, un enfant du binôme, joue à un jeu (spatialité ou numérique) ; (b) dans un second temps, ce premier enfant explique le jeu réalisé au second enfant du binôme et (c) dans un troisième temps, ce second enfant joue au jeu. Les enfants lors de la deuxième et troisième phase de l'expérimentation pouvaient être face à face ou séparés. Cette présente étude se focalise sur la deuxième phase de l'expérimentation et se concentre sur les gestes de l'enfant expliquant le jeu.

L'un des objectifs principal de cette étude est ainsi l'analyse gestuelle de l'explication chez le jeune enfant afin de voir : (a) si le type de jeu joué (jeu numérique ou jeu de spatialité) influe sur les pratiques gestuelles des enfants ; (b) si, selon que les enfants se voient, les types de gestes réalisés par les locuteurs sont différents ; il s'agit alors de tester la variable de la visibilité et (c) de voir si certains types de gestes ont plutôt comme fonction communicative principale d'aider à l'élaboration du message ou celle d'aider à la compréhension du message par l'interlocuteur.

C'est ainsi que, dans un premier temps, le cadre théorique de cette présente étude est posé et notre positionnement scientifique parmi les modèles de la multimodalité explicité. Dans un second temps, le type de séquence linguistique que nous étudions et qui semble difficilement définissable est discuté. Dans un troisième temps, la variable principale de ce papier, les unités gestuelles, est définie. S'ensuit, dans un quatrième temps, une partie consacrée à nos hypothèses théoriques. Enfin, avant de présenter les résultats et d'en discuter, la méthodologie de l'étude (présentation des participants, du protocole expérimental, du codage et des hypothèses opérationnelles) est décrite.

\section{Théorie / Positionnement}

La multimodalité dans la production et la compréhension du langage peut être considérée à différents degrés; il y aurait une version faible et une version forte de la conception de la multimodalité (Colletta, 2004, 2007). La version dite faible correspondrait aux théories Free Image Hypothesis (De Ruiter, 2000 ; Krauss, Chenet Chwala, 1996 ; Krauss, Chen et Gottesman, 2000) et Lexical Semantic Hypothesis (Butterworth et Hadar, 1989; Schegloff, 1984) dans le sens où les gestes sont certes indispensables lors de la réalisation d'un message mais la " commande motrice du mouvement est activée tardivement, bien après la conception du message verbal à transmettre » (Colletta, 2007:21). Si Krauss et al. suggèrent que le geste est initié au niveau de l'encodeur grammatical, De Ruiter pense que le geste est généré par le 
conceptualisateur (module libérant le message préverbal); il n'en reste pas moins que ces deux modèles s'accordent sur le fait: (a) que geste et langage sont des systèmes parallèles; (b) que le geste arrive tardivement dans l'élaboration du message ; (c) que le geste aide à la production et à la récupération lexicale et (d) que le geste n'est pas influencé par la structure linguistique. En revanche, pour les adeptes de la version forte de la multimodalité, les gestes aident certes la production verbale mais ils possèdent également une fonction communicative. La commande motrice du mouvement correspond à «une étape précoce du processus de production » (Colletta, 2007:21). Les gestes seraient élaborés dès la conceptualisation du message et joueraient un rôle dans l'organisation de la pensée (McNeill, 1992, 2000, 2002 ; Kita, 2000 ; Kita et Özyürek, 2003). L'Interface Hypothesis (IH) correspondrait à une version forte de la conception selon laquelle la production et la compréhension du langage seraient à considérer comme multimodaux. Selon cette hypothèse, les gestes et le langage aideraient au développement de la pensée et à la production du message par le locuteur ainsi qu'à la compréhension du message par l'interlocuteur (Kita, 2000 ; Kita et Özyürek, 2003). Les gestes proviendraient d'une interface entre gestes et parole qui serait une représentation spatio-motrice organisée dans le but de parler. Les gestes n'encoderaient pas seulement les propriétés spatio-moteurs d'un référent mais ils structureraient ces informations afin qu'elles soient compatibles avec les possibilités linguistiques d'encodage et sont déterminés par l'intention communicative générée dans le Communication Planner (Kita, 2000 ; Kita et Özyürek, 2003 ; Kita, Özyürek, Allen, Brown, Furman, Ishizuka, 2007). Les adeptes de 1'IH proposent alors un modèle basé également sur le modèle de la production orale de Levelt (1989) en y intégrant leur conception de la multimodalité ${ }^{2}$ (Figure 1).

Figure 1 : Modèle de la production multimodale selon Kita et Özyürek (2003:28)

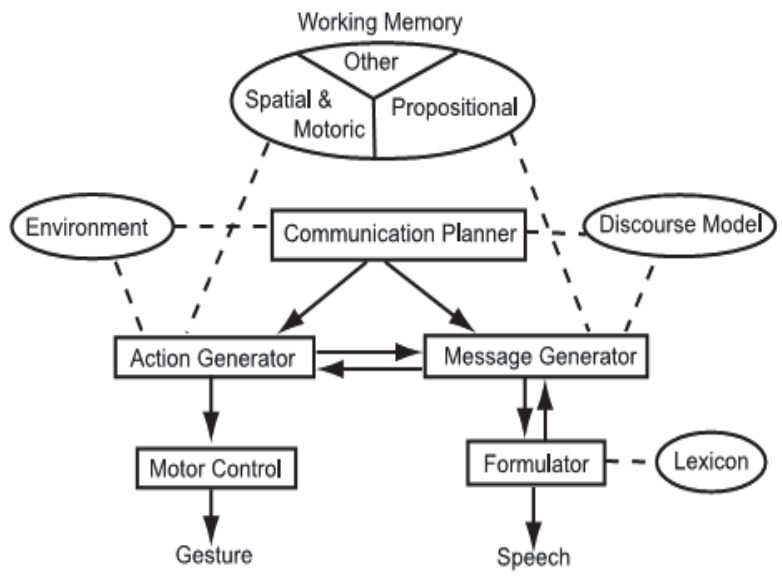

Ce qui nous intéresse davantage dans ce modèle correspond au communication planner qui génère les intentions communicatives (but du message, sélection des modalités à impliquer, etc.). Il a un accès direct (a) au discourse model, module contenant les aspects du discours : ce qui a été dit avant par les locuteurs, projection de ce qui pourra être dit, etc. et (b) à l'environment qui peut contenir des aspects tels que la visibilité des gestes de l'interlocuteur. Ainsi, le communication planner peut donner priorité à certaines informations selon le but et l'évolution du message. Ces aspects du message sont ensuite envoyés à l'action generator et au message generator.

Le message generator donne l'aspect verbal au message qui dépend du but communicatif de la situation d'énonciation et du contexte (micro-planification, dans les termes de Levelt) et qui est fortement lié au discourse model. L'action generator présente les modalités pour générer des gestes et est fortement lié à l'environment. Il apparait comme un mécanisme général de génération d'action qui peut si nécessaire être utilisé à des fins communicatives. Ainsi, l'action generator sélectionne les informations spatiales et les génère; ce processus est guidé par des schémas d'action mobilisés dans la mémoire de travail (working memory).

Le geste est alors déterminé par trois facteurs: (a) l'intention communicative ; (b) les schémas d'action et (c) le possible feed-back en temps réel entre le formulator (dont le rôle est de formuler le message), le message generator et l'action generator. En effet, comme dans le modèle de Levelt, selon Kita et Özyürek (2003), il existe une possibilité d'échange et de feedback entre le formulator et le message generator, ce dernier communiquant lui-même avec l'action generator. Ainsi, tout le long de l'élaboration d'un message, le choix des structures linguistiques évolue selon différents facteurs (par 
exemple : but communicationnel, contexte de production, interventions gestuelles de l'interlocuteur) et ceci peut également impliquer un retour sur l'élaboration des gestes. Les deux générateurs (message generator et action generator) échangent constamment des informations ce qui peut engendrer des modifications de formats. Une information spatio-moteur générée par l'action generator peut être envoyée au message generator et transformée en proposition comme une proposition générée par le message generator peut être envoyée à l'action generator et transformée en représentation spatio-moteur (Kita et Özyürek, 2003). Si l'équivalent du feeback possible entre le formulator et le message generator pour la production de formes linguistiques n'est pas possible entre le l'action generator et le motor control, selon Kita et Özyürek (2003), ce feedback se fait par le biais des échanges entre le formulator et le message generator puis entre le message generator et l'action generator. Une information traitée par le formulator comme non verbalisable peut être renvoyée au message generator, un échange a alors lieu entre le message generator et l'action generator pour voir si cette information ne peut alors pas être élaborée sous forme gestuelle.

Ce modèle est fortement inspiré de la Growth Point Theory de McNeill (1992, 2000, 2002) et McNeill et Duncan (2000) dans le sens où l'élaboration d'un message repose sur une interface entre pensée imagée et pensée linguistique : geste et parole sont des systèmes intégrés et le geste comme le langage a un rôle dans la conceptualisation du message et pas seulement dans la récupération et choix lexicaux (Alibali, Kita et Young, 2000). Chaque idée à communiquer constitue un Growth Point qui est une unité psychologique minimale, combinant imagerie et contenu linguistique, qui forment une combinaison liée temporellement et sémantiquement : geste et langage sont temporellement synchrones et co-expressifs (Kendon, 2000; McNeill, 1992; McNeill \& Duncan, 2000) et expriment nécessairement une même idée tout en pouvant introduire des aspects différents de cette idée (McNeill \& Duncan, 2000). Des deux principaux concepts de base de la Growth Point Theory en est issu l'Information Packaging Hypothesis selon laquelle les gestes aident à la conceptualisation du message en constituant une alternative communicative au langage pour encoder et organiser des informations spatiales et perceptuelles (Alibali et al, 2000 ; Kita, 2000).

L'aspect essentiel de ce modèle, pour cet article, est que le contexte de production, l'environnement, la situation de communication, etc. peuvent influencer les gestes tout comme ils influencent les choix linguistiques. Ceci semble alors être un des arguments montrant le rôle essentiel des gestes dans la conceptualisation du message.

\section{L'explication comme situation expérimentale}

L'explication est une conduite langagière qui apparait précocement dans le développement de l'enfant (Colletta et Pellenq, 2005, 2009 ; Colletta, Simon et Lachnitt, 2005 ; Veneziano et Hudelot, 2002 ; Veneziano et Sinclair, 1995). Vers 18 mois, le jeune enfant produit des conduites explicatives/justificatives (Veneziano et Hudelot, 2002). Pour produire une explication, l'enfant doit être capable : (a) d'envisager que son interlocuteur a des états internes différents de lui ; (b) de decontextualiser le langage ; (c) d'envisager que le langage peut modifier les états internes d'autrui, qui peuvent être différents des siens et (d) de produire un discours cohésifs et cohérent (Colletta et Pellenq, 2005, 2009 ; Hickmann, 2003 ; Veneziano et Hudelot, 2002). Ces compétences ne se mettent en place que progressivement (Colletta et Pellenq, 2005, 2009 ; Traxler et Gernsbacher, 1995 ; Veneziano et Hudelot, 2002) ; ce n'est pas avant 10/11 ans que l'enfant devient capable d'assurer la cohésion et la cohérence d'une production (Mazur-Palandre, 2009 ; Schneuwly, 1988). C'est ainsi que l'explication évolue selon les compétences acquises par l'enfant et que, de 2 ans à 11 ans, ce type de séquence connait une importante évolution (Colletta, Simon et Lachnitt, 2005). Ainsi, tenter de comprendre l'évolution de l'explication entre ces deux périodes du développement est un réel enjeu psycholinguistique.

L'explication est une thématique d'autant plus intéressante qu'elle est difficile à définir linguistiquement parlant. En effet, si les chercheurs ayant travaillé sur l'explication s'accordent à dire que l'explication s'organise autour d'un explanadum (phénomène ou comportement à expliquer) et d'un explanans (cause, raison, motif $=$ ce qui explique) (Colletta, 2005 ; Colletta et Pellenq, 2009 ; Lund, 2003 ; Veneziano et 
Hudelot, 2002 ; Veneziano et Sinclair, 1995), ils ne nient pas également que la polysémie du mot expliquer en fait un concept difficilement définissable. Ainsi, Grize (1990) dénombre six définitions : (a) communiquer (expliquer une idée) ; (b) développer (expliquer une maxime); (c) enseigner (expliquer une règle de jeu) ; (d) interpréter (ce livre explique bien l'œuvre de Kafka) ; (e) motiver (expliquez moi ce changement d'avis) ; (f) rendre compte (le mauvais temps explique le retard des trains). Selon Grize, l'explication peut répondre à trois questions : (a) « qu'est-ce ? » (b) « comment ? » et (c) « pourquoi ?» La plupart des études portant sur l'explication chez l'enfant se consacrent essentiellement aux explications répondant à la question «pourquoi ? : elles se concentrent alors sur les explications du type « motiver » et « rendre compte » (Colletta, 2004, 2005 ; Colletta et Pellenq, 2005, 2009 ; Lund, 2003 ; Veneziano et Hudelot, 2002 ; Veneziano et Sinclair, 1995).

Une des originalités du travail proposé est le fait que nous nous concentrerons sur un type d'explication qui n'a pas forcément été beaucoup étudié chez l'enfant : l'explication répondant à la question « comment ? » et correspondant à l'explication « enseigner » dans la typologie de Grize.

\section{Les gestes comme variables principales}

Les gestes sont catégorisables en deux grands types : les gestes autonomes (Colletta, 2004) (ou quasilinguistiques, Cosnier (2008)) et les gestes coverbaux (entre autres, Colletta, 2000, 2004, 2007, 2009 ; Colletta et al., 2009 ; Cosnier 2008 ; Kendon, 2004 ; Krauss et al., 2000 ; McNeill, 1992, 2000). Les gestes autonomes ont trois fonctions principales et sont produits aux cours d'interactions sociales : (a) les actes rituels (réalisation gestuelle d'actes sociaux tel le geste de la main pour « au revoir ») ; (b) les actes discursifs (réalisation gestuelle des actes communicationnels comme tendre le doigt strictement) et (c) les actes interactifs (au service de la synchronisation entre les locuteurs). Lorsque ces gestes sont utilisés en même temps que la version verbale du message, ils changent de statut et deviennent des gestes coverbaux. Ils ne sont, en effet, plus quasi-linguistiques au sens de Cosnier (2008), qui les définit comme « des équivalents de parole et sont parfaitement conventionnalisés selon les cultures »(Cosnier, 2008:121).

Pour ce présent travail, un intérêt tout particulier est porté sur les gestes coverbaux. Il existe un grand nombre de typologie des gestes coverbaux (entre autres, Colletta, 2000, 2004, 2007, 2009 ; Colletta et al., 2009 ; Cosnier 2008 ; Kendon, 2004 ; McNeill, 1992, 2000). Nous retenons principalement les gestes suivants :

- Le geste représentationnel : geste pouvant associer différentes parties du corps représentant (mimant) un objet concret ou une idée,

1. Il y a une balle -- Le locuteur verbalise cette clause en réalisant un rond avec ses mains.

- Le geste discursif/de structuration : geste bref structurant le discours par l'accentuation ou la mise en relief de certaines unités linguistiques,

2. C'est trop facile -- Le locuteur verbalise cette clause en faisant un mouvement de bas en haut avec la main pour insister sur l'adverbe trop.

- Le geste interactif : geste vérifiant et captant l'attention de son interlocuteur,

\section{Tourné la tête vers l'interlocuteur}

- Le geste énonciatif : geste exprimant une recherche lexicale,

\section{Mouvement de la tête vers le haut}

- Le geste de cadrage : geste exprimant un état émotionnel ou mental du locuteur,

5. C'est difficile -- Le locuteur verbalise cette clause en faisant la moue.

- Le geste performatif : réalisation non verbale d'un acte de langage non assertif (oui/non, etc.) qui renforce ou modifie la force illocutoire (Austin, 1962) lorsqu'il est verbalisé. 
- Le geste déictique : geste de pointage vers un référent présent lors de la situation d'énonciation ou indiquant sa direction,

6. C'est toi qui l'as fait -- Le locuteur verbalise ces clauses en effectuant un geste de pointage sur la personne.

\section{Hypothèses théoriques}

Dans cette section sont présentées les hypothèses théoriques de ce présent travail qui s'orientent autour de deux axes : (a) le contexte (incarné ici par le type de jeu) et (b) la condition de la passation, à savoir si les enfants se voient lors de l'explication du jeu ou pas (la variable de visibilité est ainsi testée).

\subsection{Le contexte : l'effet du type de jeu}

Les langues offrent une multitude de combinaisons possibles pour encoder un même message ; ce qui est intéressant est de savoir quels sont les critères qui génèrent la production d'un geste plutôt que d'autres. Le modèle de Kita et Özyürek (2003), comme celui de Levelt (1989) duquel ils s'inspirent, suggère l'importance de la prise en considération du contexte et de l'intention communicative. Ces modèles aident à comprendre comment l'adulte gère ces aspects multimodaux de la communication : qu'en est-il des enfants ? Afin d'aider à répondre à cette question, il a donc été décidé de faire passer aux enfants deux types de jeux : un jeu sollicitant principalement des compétences numériques et un jeu sollicitant principalement des compétences spatiales. Ce choix de deux types de jeu bien différent a été fait dans l'optique de voir : (a) si un type de jeu appelle plus de gestes et plus de matériel linguistique ou/et (b) si le types d'outils gestuels et linguistiques diffèrent selon le type de jeu. L'idée que les types de gestes varient selon la situation de production (selon le contexte, le but, la finalité du discours) va dans le sens des travaux et des modèles de Kita (2000), Kita et Özyürek (2003), McNeill (1992, 2000), Alibali et al. (2000), etc. qui plaident, non seulement en faveur d'un traitement multimodal des pratiques langagières, mais qui vont plus loin en disant que gestes et paroles sont à tout moment liés dans l'élaboration d'un message. Les gestes seraient élaborés très tôt dans la constitution d'un message et s'adapteraient aux possibilités linguistiques de la langue ; ainsi, le choix des structures linguistiques évolue selon différents facteurs (but communicationnel, contexte de production, finalité, etc.) et l'évolution de choix linguistique implique donc également un retour sur l'élaboration des gestes. Ainsi, si les gestes participent à la conceptualisation du message et s'ils aident davantage à l'encodage et à l'organisation des informations spatiales, comme le suggèrent le modèle de Kita et Özyürek (2003), la théorie du Growth Point de McNeill ou encore la Information Packaging Hypothesis, alors nous attendons des différences de taux gestuels et de types gestuels selon la tâche explicative.

Des études montrent que le type de contexte fait varier l'utilisation des gestes (Alibali et al., 2000 ; Colletta, 2004 ; Colletta et Pellenq, 2005, 2009 ; Fantazi, 2010) ainsi que les choix linguistiques (Berman, 2008 ; Jisa, 2004 ; Ravid, 2006 ; Ravid et Berman, 2010). Selon l'objet et la finalité de la situation d'énonciation, la posturo-mimo-gestualité se spécialiserait (terme de Colletta et Pellenq, 2005:6) : «les enfants " bougeraient différemment en fonction du type d'activités discursive dans lequel ils sont engagés » (Colletta et Pellenq, 2005:6). Ainsi, en production narrative et descriptive, les enfants solliciteraient davantage des gestes concrets ; en production argumentatifs, ce type de geste serait plus rare au profit des gestes interactifs ; lors d'un débat, les enfants utiliseraient plus de gestes expressifs ; enfin, en contexte explicatif (explication «en pourquoi »), les enfants accompagneraient leur production verbale de gestes abstraits et discursifs (Colletta et Pellenq, 2005, 2009). Ces résultats confirment ceux d'Alibali et al. (2000) qui avaient également trouvé que dans la tâche explicative, il y avait davantage de geste représentationnel que dans la tâche descriptive. Ainsi, selon ces résultats, les gestes ont un rôle dans la conceptualisation d'un message.

Pour notre étude, nous travaillons seulement sur l'explication mais selon les concepts mis en jeu dans l'explication (chiffre versus spatialité), les enfants conceptualisent-ils le message différemment? Est-ce 
que le type de jeu va également avoir ce type d'effet, d'autant plus que les tâches mettant en jeu l'espace semblent appeler davantage de gestes (Kita et Özyürek, 2003 ; Kita et al., 2007) ?

\subsection{La condition de passation : l'effet de la visibilité}

Il a été décidé de faire deux groupes parmi notre population : (a) un groupe où les enfants sont face à face durant tout le long de la passation (condition visibilité) et (b) un groupe où les enfants sont séparés par un rideau (condition sans visibilité). Les enfants du premier groupe se voient tout le long de la passation; ainsi le locuteur sait, lors de l'élaboration de son message, que le destinataire le voit et donc perçoit ses gestes. Les enfants du second groupe sont séparés par un rideau; ainsi le locuteur sait, lors de l'élaboration de son message, que le destinataire ne le voit et ne perçoit donc pas ses gestes.

Dans la littérature, quelques études montrent l'importance de la visibilité du destinataire sur la production gestuelle des locuteurs (Alibali, Heath et Myers, 2001 ; Bavelas, 1994 ; Bavelas, Chovil, Lawrie et Wade, 1992 ; Bavelas, Gerwing, Sutton et Prevost, 2007 ; Cohen, 1977; Cohen et Harrisson, 1973; Emmorey et Casey, 2001 ; Krauss, Dushay, Chen et Rauscher, 1995 ; Rimé, 1982). La visibilité a non seulement un effet sur la proportion des gestes mais également sur le type de gestes produits ce qui confirme la théorie de Kendon (1987) selon laquelle les conditions de transmission ont un impact aussi bien sur les unités linguistiques que sur les unités gestuelles. Ces études se sont toutes concentrées sur des populations adultes et anglophones. Il existe peu d'études ayant comme population des enfants mais elles révèlent également un effet de la visibilité sur la production de signaux visuels tels les gestes (Doherty-Sneddon et Kent, 1996), les regards et les expressions faciales (Doherty-Sneddon, McAuley, Bruce et Langton, 2000) mais ceci dépendrait de l'âge des individus : seuls les enfants les plus âgés seraient sensibles à la visibilité de leur interlocuteur. Néanmoins, ce résultat doit être interpréter prudemment dans la mesure où (a) les auteurs ont observé un seul type de geste (les gestes dits communicatifs) et (b) dans la condition séparé, les gestes ayant été faits en transgressant la séparation ont été comptabilisés (les enfants se déplaçait pour avoir dans leur champs de vision l'interlocuteur). Ainsi nous pouvons nous demander si nos jeunes enfants de 6 ans ont des comportements gestuels différents selon qu'ils aient leur interlocuteur face à eux ou qu'ils en soient séparés.

De plus, au travers des études (sur des adultes) citées précédemment, les auteurs montrent que les gestes n'ont pas tous les mêmes fonctions (Alibali et al. 2001; Bavelas, 1994 ; Bavelas et al. , 1992 ; Bavelas et al., 2007 ; Cohen, 1977; Cohen et Harrisson, 1973; Emmorey et Casey, 2001 ; Krauss et al., 1995 ; Rimé, 1982). Ceci va dans le sens d'autres analyses qui révèlent que les gestes peuvent aussi bien aider à l'élaboration du message par le locuteur qu'à la compréhension du message par l'interlocuteur adultes (Kita, 2000 ; Kita et Özyürek, 2003 ou encore Kita et al., 2007). Pouvons-nous alors parmi les gestes coverbaux, distinguer les gestes réalisés pour aider à l'élaboration du message par le locuteur et ceux davantage orienté vers le destinataire du message et donc ayant pour but de faciliter la compréhension du message par l'interlocuteur?

\section{Méthodologie}

Cette section se consacre à la description d'aspects méthodologiques. Après avoir présenté les participants de notre étude, le protocole expérimental est détaillé : la procédure, les jeux joués et les trois phases de l'expérimentation. S'ensuit la description du codage des explications. Cette partie se termine par l'explication des hypothèses opérationnelles.

\subsection{Participants}

Des enfants de CP ont été sélectionnés pour cette étude. Tous les enfants de CP de trois écoles du Nord Isère (130 élèves) ont rempli un questionnaire à partir duquel la sélection s'est faite. Tous les enfants participants sont des francophones natifs, non bilingues, ne présentant pas de trouble du comportement et d'apprentissage. Parmi tous les écoliers, 60 ont participé : 30 filles et 30 garçons (Tableau 1). 
Tableau 1. Âge des individus

\begin{tabular}{lll} 
Moyenne d'âge & Écart-type & Éventail \\
\hline 6.6 ans & 0.47 (soit 4.7 mois) & {$[6.04-7.04]$}
\end{tabular}

Les individus ont bien entendu tous été autorisés par leurs parents et ils ont participé sur la base du volontariat. La collecte des données a été faite durant les heures de classe à l'intérieur des établissements scolaires.

\subsection{Protocole expérimental}

\subsubsection{Procédure}

Il a été décidé d'analyser les pratiques langagières de jeunes enfants en situation de résolution de tâche. Deux types de tâches ont été réalisés: un jeu vidéo de chiffre et un jeu vidéo de spatialité. L'expérimentation a été réalisée en dyade. Les dyades ont été constituées par les instituteurs, qui étaient plus à même de choisir les enfants qui savent travailler ensemble. Les instituteurs assignaient à chaque enfant de la dyade un rôle : enfant-instructeur ou enfant-apprenant. L'expérimentation est constituée, comme annoncée dans l'introduction, de trois phases (ces phases sont davantage expliquées dans la section 6.2.3) :

- Phase 1. Formation de l'enfant-instructeur : l'enfant-instructeur se forme au jeu (avec le minimum d'aide des expérimentateurs afin de ne pas influencer les choix linguistiques et gestuels de l'enfant, les expérimentateurs ont des consignes strictes à respecter). Une fois le jeu réalisé, l'expérimentateur est allé chercher l'enfant-apprenant.

- Phase 2. Explication de l'enfant-instructeur à l'enfant-apprenant : l'enfant-instructeur explique le jeu à l'enfant-apprenant.

- Phase 3. Réalisation du jeu par l'enfant-apprenant sous le regard de l'enfant-instructeur : après la phase d'explication, l'enfant-apprenant réalise le jeu sous le regard de l'enfant-instructeur qui pouvait venir en aide à son camarade.

L'expérimentation s'est déroulée en deux périodes (semaine A et semaine B). Lors de la semaine A, les dyades ont réalisé un premier jeu et la semaine suivante un second jeu (l'ordre des jeux a été contrebalancé). Chacune de ces phases est davantage détaillée dans la section 6.2.3.

\subsubsection{Les jeux}

Chaque enfant a joué à deux jeux : un jeu de chiffre (La course aux nombres) et un jeu de spatialité (Le casse-brique). Le jeu de chiffre implique concentration, observation et connaissances numériques. L'enfant est face à une zone contenant 9 cartes. Sur chacune des cartes, il peut y avoir : (1) des chiffres (de 1 à 9) et (2) des formes (billes, bonbons, cerises, crayons et points). Le jeu contient 4 niveaux présentant 4 grilles différentes ainsi que des énoncés différents (6 énoncés par grilles). Un exemple de grille et d'énoncés est présenté ci-dessous (Figure 2). 
Figure 2. Grille du niveau 3 de la Course aux nombres

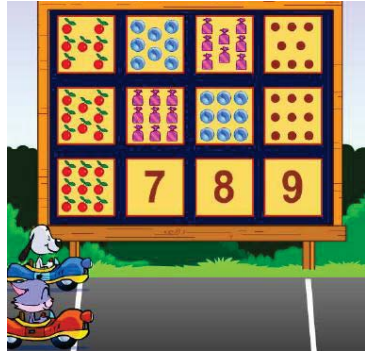

Ce jeu a plusieurs énoncés possibles (le logiciel en énonce 6 à chaque niveau) : Trouve 9 billes, Choisis 8 bonbons, Cherche 9 points, Trouve l'image qui contient le moins d'objets, Montre 8 billes, Quel chiffre vient après le 7 ?, Où est le chiffre 7 ?, Indique 8 points, 6-7-8 et ensuite?, Cherche 9 cerises. Le but du jeu est de retrouver le paquet d'objets ou le chiffre correspondant à l'énoncé. Si l'enfant pointe la bonne carte, le logiciel propose un autre énoncé. Si l'enfant échoue, le logiciel propose la même phrase jusqu'à ce que l'enfant trouve la solution. Si l'enfant ne réussit pas le niveau (à savoir donner 6 bonnes solutions avant d'arriver à 6 mauvaises solutions), le même niveau est proposé.

Le jeu de spatialité implique concentration et réflexes (Figure 3).

Figure 3. Niveau 1 du jeu Casse-briques

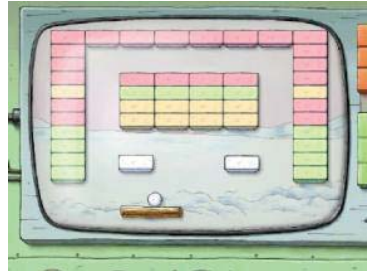

Dans ce jeu, le joueur doit renvoyer une balle à l'aide d'une raquette. Plus le temps passe, plus la balle s'accélère. Ce jeu se joue grâce à la souris. Il faut cliquer pour démarrer le jeu. Les briques à détruire sont de quatre couleurs (Figure 2) : les briques blanches se détruisent dès le premier coup; les briques jaunes se détruisent dès le premier coup et distribuent des bonus (ajout d'une balle supplémentaire, allongement de la raquette) et des malus (rétrécissement de la raquette, accélération de la balle) ; les briques vertes deviennent blanches au premier coup et disparaissent au deuxième coup ; les briques rouges deviennent vertes au premier coup, blanches au deuxième coup et disparaissent au troisième coup. Le jeu a deux niveaux : dès que l'enfant a terminé le premier, le second s'affiche.

\subsubsection{Les phases}

L'expérimentation se constitue alors de trois temps : Phase 1. Formation de l'enfant-instructeur (Figure 4) ; Phase 2. Explication de l'enfant-instructeur à l'enfant-apprenant (Figure 5) ; Phase 3. Réalisation du jeu par l'enfant-apprenant (Figure 6). Pour les deux dernières phases, deux conditions sont réalisées: une première où les enfants se voient (face-à-face) et une seconde où les enfants ne se voient pas (ils sont séparés par un rideau).

Figure 4. Schématisation de la Phase 1

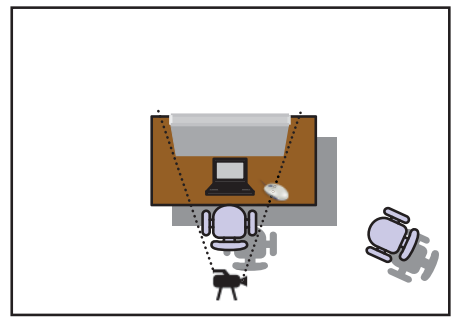

Durant la phase 1, l'enfant-instructeur se forme au jeu. Le matériel utilisé est : un miroir, un pc portable et une caméra (Figure 4). Un miroir est utilisé en plus de la caméra afin de capter le plus de gestes possible.

Durant la phase 2, l'enfant-instructeur explique le jeu à l'enfant-apprenant. Cette phase est réalisée sous les deux conditions (Figure 5). Le matériel utilisé est : un miroir, une caméra, un rideau (pour la condition sans visibilité). L'ordinateur est toujours en place mais les enfants ne s'en servent pas. C'est à cette seconde phase que cette présente étude est dédiée. 
Figure 5. Schéma de la Phase 2 (à gauche : condition visibilité / à droite : condition sans visibilité)
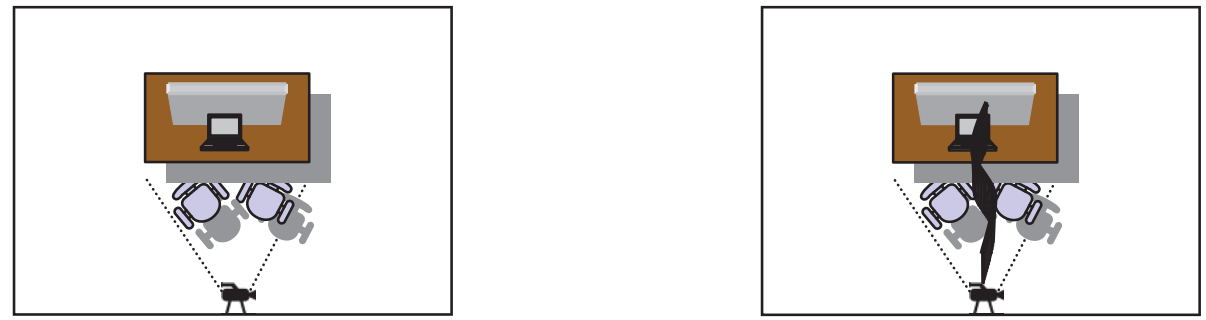

Durant la phase 3, l'enfant-apprenant joue au jeu. L'enfant-instructeur a consigne d'intervenir, s'il l'estime nécessaire, pour aider son camarade. Cette phase est réalisée sous les deux conditions (Figure 6). Le matériel utilisé est un miroir, deux caméras, un rideau (dans la condition rideau), l'ordinateur (utilisé par l'enfant-apprenant) ainsi qu'un écran (dans la condition rideau -- destiné à l'enfant-instructeur pour qu'il suive le cours du jeu de l'enfant-apprenant).

Figure 6. Schéma de la Phase 3 (à gauche : condition visibilité / à droite : condition sans visibilité)
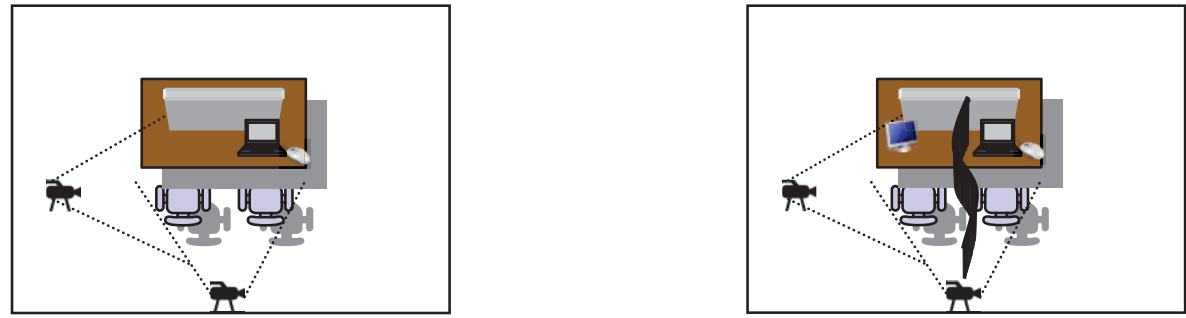

\subsection{Codage}

Le codage et la transcription des données ont été faits sous ELAN ${ }^{\mathrm{TM}}$ (http://www.lat-mpi.eu/tools/elan/). Avant de pouvoir travailler nos vidéos sous ce logiciel, un premier travail de traitement a été nécessaire. Les vidéos ont toutes été coupées (selon nos besoins) sous le logiciel Adobe ${ }^{\circledR}$ Première ${ }^{\circledR}$ Pro. Pour la phase 3 de l'expérimentation, deux caméras étaient utilisées ; il a donc été utile de synchroniser les deux vidéos. Ensuite, le son des vidéos a été extrait. Ainsi, nous avions les deux fichiers utiles à ELAN : un fichier vidéo et un fichier son. Le travail sous ELAN s'est déroulé en deux phases : la phase de transcription et celle de codage.

Pour le présent travail, les gestes ont été codés. Pour identifier les unités gestuelles qu'il s'apprête à annoter, le codeur prend en compte deux principaux critères - le mouvement et l'emplacement -auxquels il attribue une valeur entre 0 et 2 (Tableau 2). Un troisième critère est celui de la configuration ce dernier n'est utilisable que pour les gestes concrets. Le mouvement est identifié comme un geste lorsque la somme des valeurs attribuées pour les deux premiers critères est strictement supérieure à 2 . Le critère de la configuration ajoute 1 ou 2 points aux gestes concrets selon son degré de précision dans la représentation d'une forme. 
Tableau 2. Identification d'un geste

\begin{tabular}{|c|c|c|}
\hline Critères & Échelle & Codage \\
\hline \multirow[t]{3}{*}{ Mouvement } & Repérable (bonne amplitude) & 2 \\
\hline & Peu repérable & 0 \\
\hline & Entre les deux & 1 \\
\hline \multirow[t]{3}{*}{ Emplacement } & Dans l'espace frontal du locuteur & 2 \\
\hline & Sur un côté (peu ou pas repérable par l'interlocuteur) & 0 \\
\hline & Entre les deux & 1 \\
\hline \multirow[t]{3}{*}{ Configuration } & Correspond à une forme précise & 2 \\
\hline & Correspond à une forme imprécise & 0 \\
\hline & Entre les deux & 1 \\
\hline
\end{tabular}

Seuls les gestes des mains et de la tête sont pris en considération. Pour chaque geste, une valeur lui a été ensuite attribuée. Rappelons que le geste pouvait avoir diverses valeurs présentées dans la section 4 : représentationnel, discursif, interactif, énonciatif, de cadrage, performatif et déictique. Une procédure de méthode de juges est en cours pour vérifier la réplicabilité de notre méthode de codage.

\subsection{Hypothèses opérationnelles}

Les figures ci-dessous résument nos attentes quant aux hypothèses théoriques présentées dans la section 5 et explicitent leurs liens avec les hypothèses opérationnelles.

Figure 7. Hypothèse théorique 1 / hypothèse opérationnelle $1 \mathrm{a}$ et $1 \mathrm{~b}$

Les gestes sont activés précocement tout comme le langage et participent à la conceptualisation du message.

\section{Hypothèse théorique 1}

Selon le TYPE DE JEU à réaliser, les choix des gestuels des enfants sont différents.

\section{Hypothèses opérationnelles 1a et $1 \mathrm{~b}$}

1a. Le nombre de gestes varie selon le type de jeu : un taux plus important de gestes lors de l'explication du jeu de spatialité est attendu.

1b. Le type de gestes varie selon le type de jeu : un taux plus important de gestes représentationnels lors de l'explication du jeu de spatialité est attendu.

Figure 8 . Hypothèse théorique 2 / hypothèse opérationnelle $2 \mathrm{a}$ et $2 \mathrm{~b}$

Théorie
Si les gestes aident à la compréhension comme à la production du langage, les individus produisent plus
ou moins de gestes sans interlocuteurs directs. De plus, il est possible de séparer les deux fonctions du
geste : le geste pour le locuteur (qui aide à l'élaboration d'un message) versus le geste pour le destinataire
(qui aide à la compréhension).
Hypothèse théorique $\mathbf{2}$
Selon la CONDITION, les choix des gestuels des enfants sont différents.
Hypothèses opérationnelles 2a et $\mathbf{2 b}$
2a. Le nombre de gestes varie selon la condition : un taux plus important de gestes lors des explications
lorsque les enfants se voient est attendu.
2b. Le type de gestes varie selon la condition : un taux plus important de gestes interactifs et déictiques
est attendu lorsque les enfants se voient.




\section{Résultats}

Selon le plan expérimental S15<CONDITION $2>*$ JEU 2, où S représente le facteur aléatoire sujet, CONDITION est le facteur inter sujets (à deux modalités : face-à-face ou séparés) et JEU représente le facteur intra-sujet (à deux modalités : jeu de spatialité et jeu de chiffre - tous les individus ont donc passé les deux jeux), 30 dyades réparties en deux groupes (15 dyades réalisant l'expérimentation en condition visibilité et 15 en condition sans visibilité) ont participé. Rappelons que les analyses qui suivent portent sur l'explication de l'enfant-instructeur à l'enfant-apprenant lors de la phase 2 de l'expérimentation.

Afin de tester nos hypothèses, plusieurs analyses ont été effectuées. Des analyses de variance ${ }^{3}$ à plusieurs facteurs ont été lancées pour différentes variables dépendantes : le nombre de gestes total, le nombre de gestes représentationnels, le nombre de gestes discursifs, le nombre de gestes interactifs, le nombre de gestes énonciatifs, le nombre de gestes de cadrage, le nombre de gestes performatifs et enfin le nombre de gestes déictiques. Notons que les analyses pour les variables nombre de gestes de cadrage et nombre de gestes performatifs ne sont pas présentées dans la mesure où les proportions sont trop faibles pour lancer un test d'analyse de variance (ces types de gestes n'apparaissent pas dans les quatre conditions (Jeu de spatialité condition Sans visibilité / Jeu de spatialité condition Visibilité / Jeu de Chiffre condition Visibilité / Jeu de Chiffre condition Sans Visibilité). Enfin, pour chacune des analyses de variance aucune interaction entre les deux facteurs de notre étude (JEU et CONDITION) n'est significative.

\subsection{Le nombre de gestes}

Une analyse de variance a donc été faite sur le nombre total de gestes par explication en jeu de spatialité et de chiffre et dans les deux conditions. Les résultats révèlent que le facteur JEU a un effet significatif sur le nombre de gestes dans l'explication $\left(F_{(1,28)}=4,801, \mathrm{p}=0,037\right)$. Les analyses révèlent que les enfants de notre étude produisent davantage de gestes lorsqu'ils expliquent le jeu de spatialité que quand ils expliquent le jeu de chiffre (Tableau 1a). En revanche, le facteur CONDITION n'a pas d'effet significatif (Tableau 1b).

Tableau 1a. Comparaison du nombre de geste selon le facteur JEU

\begin{tabular}{lllllll}
\hline & & $\mathrm{n}=$ & $\bar{x}$ & $F$ & Valeur de $\mathrm{p}=$ & $<0,05=*$ \\
\hline \multirow{2}{*}{ JEU } & Spatialité & 30 & 6,23 & 4,801 & 0,037 & $*$ \\
\cline { 2 - 4 } & Chiffre & 30 & 3,93 & & & \\
\hline
\end{tabular}

Tableau 1b. Comparaison du nombre de geste selon le facteur CONDITION

\begin{tabular}{lllllll}
\hline \multirow{2}{*}{ CONDITION } & $\mathrm{n}=$ & $\bar{x}$ & $F$ & Valeur de $\mathrm{p}=$ & $<0,05=*$ \\
& Visibilité & 15 & 6,16 & 2,027 & 0,166 & $\begin{array}{l}\text { Non } \\
\text { significatif }\end{array}$ \\
\cline { 2 - 5 } & Sans visibilité & 15 & 4,00 & & & \\
\hline
\end{tabular}

Ces premiers résultats confirment en partie nos hypothèses opérationnelles (HO) 1a et 2a. Nous attendions une proportion plus importante de gestes selon le type de jeu expliqué et plus précisément nous pensions qu'il aurait plus de gestes dans les explications du jeu de spatialité que dans les explications du jeu de chiffre (HO 1a). Cette première hypothèse est validée par nos résultats. En revanche, l'hypothèse HO 2 b selon laquelle le nombre de gestes varie significativement selon la condition de passation (une proportion plus importante de geste était attendue dans les explications d'enfants voyant leur interlocuteur) n'est pas validé : la différence de proportion de gestes selon la condition de passation n'est pas significative.

\subsection{Les gestes représentationnels}

Une analyse de variance a été réalisée sur le nombre de gestes représentationnels par explication en jeu de spatialité et de chiffre et dans les deux conditions. Les résultats révèlent que le facteur JEU a un effet significatif sur le nombre de gestes représentationnels dans l'explication $\left(F_{(1,28)}=12,003, \mathrm{p}=0,002\right)$. Les 
analyses révèlent que les enfants de notre étude produisent davantage de gestes représentationnels lorsqu'ils expliquent le jeu de spatialité que quand ils expliquent le jeu de chiffre (Tableau 2a). En revanche, le facteur CONDITION n'a pas d'effet significatif (Tableau 2b).

Tableau 2a. Comparaison du nombre de gestes représentationnels selon le facteur JEU

\begin{tabular}{lllllll}
\hline & & $\mathrm{n}=$ & $\bar{x}$ & $F$ & Valeur de $\mathrm{p}=$ & $<0,05=*$ \\
\hline \multirow{2}{*}{ JEU } & Spatialité & 30 & 2.93 & 12,003 & 0,002 & $*$ \\
\cline { 2 - 4 } & Chiffre & 30 & 0.9 & & & \\
\hline
\end{tabular}

Tableau 2b. Comparaison du nombre de gestes représentationnels selon le facteur CONDITION

\begin{tabular}{lllllll}
\hline & & $\mathrm{n}=$ & $\bar{x}$ & $F$ & Valeur de $\mathrm{p}=$ & $<0,05=*$ \\
\hline \multirow{2}{*}{ CONDITION } & Visibilité & 15 & 2,2 & 0,635 & 0,432 & $\begin{array}{l}\text { Non } \\
\text { significatif }\end{array}$ \\
\cline { 2 - 4 } & Sans visibilité & 15 & 1,63 & & &
\end{tabular}

Ces résultats confirment en partie nos hypothèses opérationnelles (HO) $1 \mathrm{~b}$ et $2 \mathrm{~b}$. Nous attendions une proportion plus importante de certains types de gestes selon le type de jeu expliqué et la condition de passation. Le résultat du tableau $2 \mathrm{a}$ valide $\mathrm{HO} 1 \mathrm{~b}$ : la proportion des gestes représentationnels est significativement plus importante dans les explications du jeu de spatialité que dans les explications du jeu de chiffre. En revanche, le résultat du tableau $2 b$ révèle que la condition de passation n'a pas d'effet significatif sur la proportion des gestes représentationnels.

\subsection{Les gestes discursifs}

Une analyse de variance a été réalisée sur le nombre de gestes discursifs par explication en jeu de spatialité et de chiffre et dans les deux conditions. Les résultats révèlent que les facteurs JEU (Tableau 3a) et CONDITION n'ont pas d'effet significatif (Tableau 3b).

Tableau 3a. Comparaison du nombre de gestes discursifs selon le facteur JEU

\begin{tabular}{lllllll}
\hline & & $\mathrm{n}=$ & $\bar{x}$ & $F$ & Valeur de $\mathrm{p}=$ & $<0,05=*$ \\
\hline \multirow{2}{*}{ JEU } & Spatialité & 30 & 1,83 & 0,289 & 0,595 & Non \\
\cline { 2 - 5 } & Chiffre & 30 & 1,53 & & & Significatif \\
\hline
\end{tabular}

Tableau 3b. Comparaison du nombre de gestes discursifs selon le facteur CONDITION

\begin{tabular}{|c|c|c|c|c|c|c|}
\hline & & $\mathrm{n}=$ & $\bar{x}$ & $F$ & Valeur de $\mathrm{p}=$ & $<0,05=*$ \\
\hline \multirow[t]{2}{*}{ CONDITION } & Visibilité & 15 & 2 & \multirow[t]{2}{*}{0,918} & \multirow[t]{2}{*}{0,346} & Non \\
\hline & Sans visibilité & 15 & 1,37 & & & Significatif \\
\hline
\end{tabular}

Ces résultats infirment nos hypothèses ( $\mathrm{HO} 1 \mathrm{~b}$ et $2 \mathrm{~b}$ ) dans la mesure où nous attendions un effet du type de jeu à expliquer et de la condition de passation sur la proportion de gestes selon leur type: les différences de proportion des gestes discursifs selon le jeu à expliquer et la condition de passation ne sont pas significatives.

\subsection{Les gestes interactifs}

Une analyse de variance a été réalisée sur le nombre de gestes interactifs par explication en jeu de spatialité et de chiffre et dans les deux conditions. Les résultats révèlent que le facteur JEU n'a pas d'effet significatif (Tableau 4a). En revanche, le facteur CONDITION a un effet significatif sur le nombre de gestes interactifs dans l'explication $\left(F_{(1,28)}=4,171, \mathrm{p}=0,05\right)$. Les analyses révèlent que les enfants de notre étude produisent davantage de gestes interactifs lorsqu'ils voient leur interlocuteur quand lorsqu'ils ne les voient pas (Tableau 4b).

Tableau 4a. Comparaison du nombre de gestes interactifs selon le facteur JEU

$\begin{array}{llll}\mathrm{n}= & \bar{x} & \text { Valeur de } \mathrm{p}=\quad<0,05=*\end{array}$




\begin{tabular}{lllllll}
\hline \multirow{2}{*}{ JEU } & Spatialité & 30 & 0,56 & 0,879 & 0,356 & $\begin{array}{l}\text { Non } \\
\text { Significatif }\end{array}$ \\
\cline { 2 - 4 } & Chiffre & 30 & 0,73 & & & S \\
\hline
\end{tabular}

Tableau 4b. Comparaison du nombre de gestes interactifs selon le facteur CONDITION

\begin{tabular}{lllllll}
\hline & & $\mathrm{n}=$ & $\bar{x}$ & $F$ & Valeur de $\mathrm{p}=$ & $<0,05=*$ \\
\hline \multirow{2}{*}{ CONDITION } & Visibilité & 15 & 0,93 & 4,171 & 0,05 & $*$ \\
\cline { 2 - 4 } & Sans Visibilité & 15 & 0,36 & & & \\
\hline
\end{tabular}

Ces résultats confirment en partie nos hypothèses opérationnelles (HO) $1 \mathrm{~b}$ et $2 \mathrm{~b}$. Nous attendions une proportion plus importante de certains types de gestes selon le type de jeu expliqué et la condition de passation. Le résultat du tableau 4a ne valide pas notre hypothèse : la différence de proportion des gestes interactifs dans les explications du jeu de spatialité et dans les explications du jeu de chiffre n'est pas significative. En revanche, le résultat du tableau $4 \mathrm{~b}$ révèle que la condition de passation a un effet significatif sur la proportion des gestes interactifs : la proportion des gestes interactifs est plus importante dans les explications en condition visibilité.

\subsection{Les gestes énonciatifs}

Une analyse de variance a été réalisée sur le nombre de gestes énonciatifs par explication en jeu de spatialité et de chiffre et dans les deux conditions. Les résultats révèlent que les facteurs JEU (Tableau 5a) et CONDITION n'ont pas d'effet significatif (Tableau 5b).

Tableau 5a. Comparaison du nombre de gestes interactifs selon le facteur JEU

\begin{tabular}{|c|c|c|c|c|c|c|}
\hline & & $\mathrm{n}=$ & $\bar{x}$ & $F$ & Valeur de $p=$ & $<0,05=*$ \\
\hline \multirow[t]{2}{*}{ JEU } & Spatialité & 30 & 0,73 & \multirow[t]{2}{*}{0,046} & \multirow[t]{2}{*}{0,832} & \\
\hline & Chiffre & 30 & 0,60 & & & Significatif \\
\hline
\end{tabular}

Tableau 5b. Comparaison du nombre de gestes interactifs selon le facteur CONDITION

\begin{tabular}{lllllll}
\hline & & $\mathrm{n}=$ & $\bar{x}$ & $F$ & Valeur de $\mathrm{p}=$ & $<0,05=*$ \\
\hline \multirow{2}{*}{ CONDITION } & Visibilité & 15 & 0,77 & 0,529 & 0,473 & $\begin{array}{l}\text { Non } \\
\text { significatif }\end{array}$ \\
\cline { 2 - 4 } & Sans visibilité & 15 & 0,57 & & & . \\
\hline
\end{tabular}

Ces résultats infirment nos hypothèses $(\mathrm{HO} 1 \mathrm{~b}$ et $2 \mathrm{~b}$ ) dans la mesure où nous attendions un effet du type de jeu à expliquer et de la condition de passation sur la proportion de gestes selon leur type : les différences de proportion des gestes énonciatifs selon le jeu à expliquer et la condition de passation ne sont pas significatives.

\subsection{Les gestes déictiques}

Une analyse de variance a été réalisée sur le nombre de gestes déictiques par explication en jeu de spatialité et de chiffre et dans les deux conditions. Les résultats révèlent que le facteur JEU n'a pas d'effet significatif (Tableau 6a). En revanche, le facteur CONDITION a un effet significatif sur le nombre de gestes déictiques $\left(F_{(1,28)}=4,375, \mathrm{p}=0,046\right)$. Les analyses révèlent que les enfants de notre étude produisent davantage de gestes déictiques lorsqu'ils expliquent les jeux en condition visibilité (Tableau 6b).

Tableau 6a. Comparaison du nombre de gestes déictiques selon le facteur JEU

\begin{tabular}{lllllll}
\hline & & $\mathrm{n}=$ & $\bar{x}$ & $F$ & Valeur de $\mathrm{p}=$ & $<0,05=*$ \\
\hline \multirow{2}{*}{ JEU } & Spatialité & 30 & 0,13 & 3,5 & 0,072 & $\begin{array}{l}\text { Non } \\
\text { significatif }\end{array}$ \\
\cline { 2 - 5 } & Chiffre & 30 & 0,03 & & & \\
\hline
\end{tabular}


Tableau 6b. Comparaison du nombre de gestes déictiques selon le facteur CONDITION

\begin{tabular}{lllllll}
\hline \multirow{2}{*}{ CONDITION } & \multicolumn{1}{c}{$\mathrm{n}=$} & $\bar{x}$ & $F$ & Valeur de $\mathrm{p}=$ & $<0,05=*$ \\
\cline { 2 - 6 } & Visibilité & 15 & 0,167 & 4,375 & 0,046 & $*$ \\
\cline { 2 - 4 } & Sans visibilité & 15 & 0 & & & \\
\hline
\end{tabular}

Ces résultats confirment en partie nos hypothèses opérationnelles (HO) $1 \mathrm{~b}$ et $2 \mathrm{~b}$. Nous attendions une proportion plus importante de certains types de gestes selon le type de jeu expliqué et la condition de passation. Le résultat du tableau 6 a ne valide pas notre hypothèse : la différence de proportion des gestes déictiques dans les explications du jeu de spatialité et dans les explications du jeu de chiffre n'est pas significative. En revanche, le résultat du tableau $6 \mathrm{~b}$ révèle que la condition de passation a un effet significatif sur la proportion des gestes déictiques: leur proportion est plus importante dans les explications en condition visibilité.

\section{Discussion}

Le but de ce papier était de voir si les gestes, dans un même type de séquence linguistique, l'explication " en comment », variaient selon le type de jeu à expliquer et selon la présence directe (les locuteurs sont face à face) ou indirecte (les locuteurs ne se voient pas) des locuteurs. Ainsi les individus réalisent-ils des gestes coverbaux différents selon les situations ? Les gestes aident-ils à la conceptualisation du message ? Pour répondre à cette problématique, notre protocole expérimental impliquait alors un facteur intraindividuel JEU (se déclinant en deux modalités: jeu de spatialité et jeu de chiffre) et un facteur interindividuel CONDITION (se déclinant en deux modalités : face à face ou séparés). Tous les enfants ont donc expliqué les deux types de jeu. Les hypothèses de départ sont alors que le nombre de gestes varie selon le type de jeu à expliquer et la condition dans laquelle les individus réalisent l'explication. De plus, le type de gestes que les enfants font lors de leur explication sont différents selon les deux facteurs JEU et CONDITION. Des effets des variables du contexte et de visibilité sont révélés par les analyses.

\subsection{Les gestes : influencés par le contexte (type de jeu à expliquer) ?}

Les analyses de variance réalisées pour ce présent papier révèlent que deux variables dépendantes varient selon le facteur intra-sujet JEU : nombre total de gestes et nombre de gestes représentationnels. En effet, lors de l'explication du jeu de spatialité les enfants de notre expérimentation produisent significativement plus de gestes que lors de l'explication du jeu de chiffre (première analyse de variance, section 7.1). Ceci va dans le sens des travaux sur des populations adultes, entre autres, de Kita (2000), Kita et Özyürek (2003) ou encore Kita et al. (2007), selon lesquelles un contexte communicationnel impliquant davantage de concepts de spatialités engendrerait la production davantage de gestes lors de l'élaboration du message verbal. Ainsi, les jeunes enfants de notre étude suivent cette tendance en produisant davantage de gestes dans le contexte le plus spatial. De plus, lorsque nous affinons les analyses en regardant les types de gestes, nous notons que seule la proportion des gestes représentationnels varie significativement selon le type de jeu expliqué (seconde analyse de variance, section 7.2). En effet, lors de l'explication du jeu de spatialité les enfants de notre expérimentation produisent significativement plus de gestes représentationnels que lors de l'explication du jeu de chiffre. Ce jeu impliquant des concepts concrets de spatialité et de mouvements incite alors les enfants à utiliser davantage ce type de gestes concrets. Nos analyses vont dans le sens des travaux d'Alibali et al. (2000) dont l'analyse révèle que les enfants font davantage des gestes représentationnels dans les explications que dans les descriptions qui ne mettent pas autant en jeu des notions de spatialités. Ces premiers résultats suggèrent que les gestes aident à la conceptualisation du message dans la mesure où ils ont un taux différent selon que les tâches explicatives impliquent plus ou moins de concepts de spatialités. Ceci confirme alors le modèle de Kita et Özyürek (2003) et la théorie du Growth Point de McNeill : le module responsable de la génération des gestes (action generator) est influencé par l'environment et le discourse model. Ainsi, en suivant la logique du modèle de Kita et Özyürek (2003), l'élaboration des gestes semble être une étape précoce de la 
production langagière tout aussi sensible que les formes linguistiques à l'environment et au discourse model, conclusion également faite par Alibali, et al (2000). L'illustration 1 est un exemple de geste représentationnel.

Illustration 1. Dyade 01 -- Condition sans visibilité, Jeu de spatialité

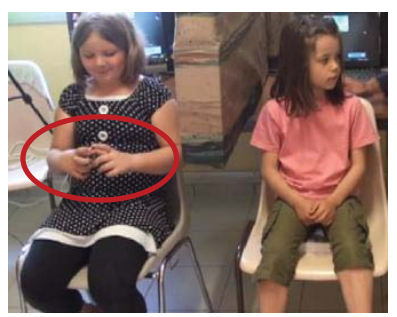

Cette illustration provient d'une explication du jeu de spatialité dans la condition sans visibilité. L'enfant-instructeur (à gauche) explique le jeu à l'enfant-apprenant (à droite). Au début de son explication, elle introduit l'idée de la balle linguistiquement et forme un rond avec ses mains représentant cette balle.

\subsection{Les gestes: influencés par la situation de communication (condition de passation)?}

Les analyses de variance réalisées pour ce présent papier révèlent que deux variables dépendantes varient selon le facteur inter-sujet CONDITION (visibilité ou absence de visibilité): la proportion des gestes interactifs et celle des gestes déictiques. En effet, les gestes interactifs ont une proportion plus importante dans les explications lorsque les enfants sont face à leur interlocuteur que lorsqu'ils en sont séparés (quatrième analyse de variance, section 7.4). Ce type de geste, par définition, tend à vérifier et à capter l'attention de l'interlocuteur. Ainsi, le canal visuel étant coupé dans la condition sans visibilité, les enfants produisent très peu ce type de geste orienté principalement vers l'interlocuteur. Ce résultat laisse à penser que ce type de geste est réalisé davantage pour l'interlocuteur que pour le locuteur lui-même. En effet, des travaux (Alibali et al., 2001 ; Kita, 2000 ; Kita et Özyürek, 2003, Kita et al., 2007 ; Lozano et Tversky, 2006) montrent que les gestes peuvent aussi bien aider à l'élaboration du message par le locuteur que la compréhension du message par l'interlocuteur. Nous pouvons alors penser que le geste interactif est davantage produit pour l'interlocuteur que pour le locuteur. L'illustration 2 est un exemple de geste interactif.

Illustration 2. Dyade 02 -- Condition visibilité, Jeu de spatialité

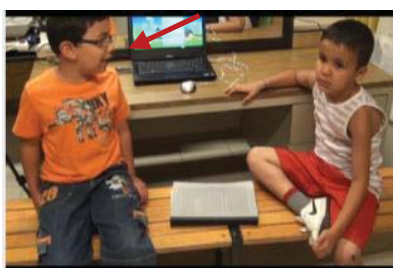

Cette illustration provient d'une explication du jeu de spatialité dans la condition visibilité. L'enfant-instructeur (à gauche) explique le jeu à l'enfant-apprenant (à droite). L'enfant-instructeur explique le jeu et au bout de quelques secondes, il fait un geste de la tête vif en direction de l'enfant-apprenant afin de vérifier et/ou capter son attention.

De plus, il s'avère que les gestes déictiques sont plus nombreux dans les explications quand les enfants sont face à leur interlocuteur que quand ils en sont séparés (sixième analyse de variance, section 7.6). Les gestes déictiques, par définition, sont des gestes de pointage vers un référent présent lors de la situation d'énonciation ou indiquant sa direction. Dans la mesure où ce type de geste est totalement absent de la condition sans visibilité, nous pouvons nous demander si ce type de geste n'aurait pas la même fonction que le geste interactif : un geste davantage centré sur le destinataire du message afin de faciliter la compréhension du message. L'illustration 3 est un exemple de geste déictique. 
Illustration 3. Dyade 12 -- Condition visibilité, Jeu de spatialité

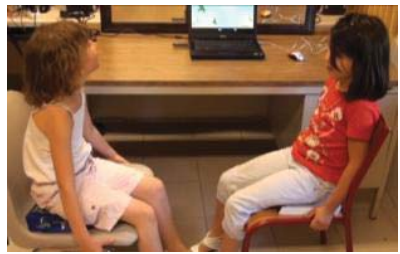

Cette illustration provient d'une explication du jeu de spatialité dans la condition visibilité. L'enfant-instructeur (à gauche) explique le jeu à l'enfant-apprenant (à droite). L'enfant-instructeur explique le jeu et au bout de quelques secondes, il verbalise « et avec la souris » et en même temps fait un geste de la tête en pointant son menton sur la souris de l'ordinateur.

Nos résultats ne vont pas totalement dans le sens de la seule étude (à notre connaissance) se concentrant sur l'effet de la visibilité de l'interlocuteur sur la production des gestes chez les jeunes enfants. DohertySneddon et Kent (1996) ont travaillé sur les gestes communicatifs. Bien qu'il y ait quelques problèmes méthodologiques avec cette précédente étude, les résultats révèlent qu'à l'âge de 6 ans, la production de gestes n'est pas sensible à la visibilité ; en revanche, à 11 ans, c'est le cas : les enfants de 11 ans produisent plus de gestes communicatifs lorsqu'ils sont face à l'interlocuteur. Les auteurs en conclut que les enfants de 6 ans ont besoin des gestes pour faciliter l'élaboration du message et ce peu importe la condition de passation (face à face ou séparés) alors que les enfants de 11 ans s'adaptent à la situation : quand ils ne voient pas leur interlocuteur, ils font alors moins de gestes et compenseraient l'absence de geste par du verbal. Nos analyses vont plus loin en révélant que l'effet de la visibilité sur la production des gestes ne dépend pas seulement de l'âge des individus mais aussi des types de gestes observés et de la tâche à effectuer.

\subsection{Quelle fonction pour quel type de geste?}

Ces analyses mettent en lumière le comportement gestuel de jeunes enfants lors d'une tâche explicative en «comment». Les résultats montrent que certains gestes sont produits davantage dans la condition visibilité : les gestes interactifs et déictiques ; d'autres sont davantage produits selon le jeu à expliquer : les gestes représentationnels, plus présents dans les explications du jeu de spatialité et enfin d'autres (les gestes discursifs et énonciatifs) ne sont sensibles ni au contexte ni à la situation d'énonciation. Ces résultats engendrent la question suivante : pouvons-nous dire que certains gestes sont plus orientés vers le locuteur (et donc plus à même à faciliter l'élaboration du message), que d'autres gestes sont davantage orientés vers l'interlocuteur (et donc plus à même à faciliter la compréhension du message) ou encore que d'autres ont une double fonction: faciliter l'élaboration du message et sa compréhension par l'interlocuteur? Bien entendu, pour répondre à une telle question des analyses complémentaires seraient à envisager. Néanmoins, nous nous risquons à proposer une réponse concernant les jeunes enfants de notre étude : (a) les gestes interactifs et déictiques seraient des gestes principalement produits afin de faciliter la compréhension du message par l'interlocuteur ; (b) les gestes représentationnels, discursifs et énonciatifs auraient une double fonction : faciliter l'élaboration du message et sa compréhension par l'interlocuteur. En effet, ces types de gestes sont aussi bien présents lorsque les locuteurs sont face à face ou séparés. Ainsi, les gestes peuvent à la fois aider à la production du message (comme le suggèrent, entre autres Goldin-Meadow, 2003) qu'être centrés vers le destinataire afin de l'aider dans sa compréhension du message (comme le suggèrent, entre autres Beattie et Shovelton, 2000, 2002 ; Clark, 1996) voire orientés à la fois vers le locuteur et le destinataire (comme le suggère, entre autres Alibali et al., 2001; Kita, 2000 ; Kita et Özyürek, 2003 ; Kita et al., 2007 ; Lozano et Tversky, 2006) : la fonction du geste dépend alors de sa nature (représentationnel, interactif, déictique, etc.).

D'autres auteurs avant nous, se concentrant sur l'analyse de production d'adultes, en sont arrivés à une conclusion similaire selon laquelle la visibilité aurait un effet sur la production des gestes (Alibali et al., 2001 ; Bavelas, 1994 ; Bavelas et al., 1992 ; Bavelas et al., 2007 ; Cohen, 1977; Cohen et Harrisson, 1973; Emmorey et Casey, 2001 ; Krauss et al., 1995 ; Rimé, 1982) : le locuteur ne fait pas les mêmes gestes et ne produit pas une même proportion de gestes selon qu'il est face à son interlocuteur ou qu'il ne l'est pas. Les adultes, lorsque s'ils sont face à face avec leur interlocuteur : (a) produisent plus des gestes « illustratifs » dans une tâche de donner des directions spatiales (Cohen, 1977; Cohen et Harrisson, 1973); (b) produisent plus de gestes «communicatifs » lors de tâches d'opinion sur des films (Rimé, 
1982) ; (c) font plus de gestes «intertactifs » lors d'une tâche de narration personnelle (Bavelas et al., 1992); (d) ont une proportion plus importante de gestes lors d'une tâche de descriptions de graphiques (Krauss et al., 1995) ; (e) produisent plus de gestes représentationnels lors d'une tâche de rappel d'un dessin-animé (Alibali et al., 2001) ou (f) produisent d'une manière général plus de gestes (Emmorey \& Casey, 2001) lorsqu'ils sont séparés de leurs interlocuteurs (que ce soit par un moyen technologique comme l'interphone telles les études de Cohen (1977), Cohen et Harrisson (1973), Krauss et al. (1995) ou par un rideau (ou autre) telles les études de Alibali et al. (2001), Bavelas et al. (1992), Emmorey \& Cassey (2001), Rimé (1982)). Néanmoins, il n'y a pas de consensus ; en effet, les études confrontant différents types de gestes dans leurs analyses montrent que certains gestes ne varient pas d'une modalité à l'autre tels que les gestes topics de l'étude de Bavelas et al. (1992) ou les beats de l'étude de Alibali et al. (2001). Les travaux de Bavelas (Bavelas, 1994 ; Bavelas et al., 1992 ; Bavelas et al., 1992) et d'Alibali et al. (2001) sont particulièrement intéressants, malgré une tâche bien différente de la nôtre dans la mesure où : (a) ils traitent approximativement des mêmes types de gestes que ceux traités dans notre étude et (b) ils ont des conditions de passation quasi similaire aux nôtres (face-à-face ou locuteurs séparés par un rideau, un écran ou un paravent) et (c) ils introduisent l'idée de comparer différents types de gestes (et donc l'idée que certains gestes sont sensibles à la variable de visibilité et d'autres non). La conclusion des travaux de Bavelas est que les locuteurs adultes produisent davantage de gestes interactifs dans une condition de face-à-face que dans une condition où ils ne voient pas leur interlocuteur ; néanmoins, ils produisent autant de gestes topic (qui peuvent être assimilés aux gestes représentationnels) dans les deux conditions. Les résultats de ce présent papier montrent que les enfants de notre étude ont un comportement similaire. Néanmoins, les travaux d'Alibali et al. (2001) révèlent le contraire : les gestes représentationnels ont une proportion plus importante dans les interactions en face-à-face que dans les interactions où les locuteurs adultes sont séparés visuellement alors que les beats sont produits dans une proportion similaire dans les deux conditions. Une des raisons pour laquelle les enfants de notre étude font autant de gestes représentationnels dans les deux conditions de passation (face à face et séparé) pourrait être que le facteur JEU serait un aspect du contexte plus fort que la condition de passation (visibilité ou non de l'interlocuteur). Le jeu de spatialité, imposant énormément de concepts spatiaux, contraindrait tellement l'enfant qu'en condition séparé il produirait tout de même ce type de geste. Ainsi, il est bien difficile de poser un consensus dans la mesure où tous ses travaux impliquent des tâches différentes (narration, explication, etc.), des classifications différentes (gestes topic / gestes représentationnels, etc.) et sans doute à des identifications différentes.

Pour finir, l'étude des gestes lors de l'explication en «comment» montre également que ces jeunes enfants ne possèdent pas encore tout le panel de gestes pouvant être retrouvé dans des productions d'enfants plus âgés ou d'adultes (Colletta, 2004 ; Colletta et Pellenq, 2005, 2009 ; Fantazi, 2010). En effet, seulement un ou deux individus de notre population a produit des gestes de cadrage ou des gestes performatifs.

\section{Perspectives}

Ce présent travail est le premier d'une longue série. En effet, il s'agit des toutes premières analyses du projet PAMEALE (cf. note de fin 1).

Une première perspective sera notamment d'analyser le lien langage verbal et langage gestuel de la phase 2 de notre protocole expérimental.

Une seconde perspective se concentrera davantage sur la phase 3 en se focalisant sur l'étude de l'aspect collaboratif et de la qualité du guidage selon les conditions.

Ces premiers résultats et les futurs permettront alors de dresser une modélisation de l'explication « en comment » chez le jeune enfant. Néanmoins, afin de compléter le tableau et afin de pouvoir obtenir une modélisation la plus réaliste possible, ce projet devra être complété par d'autres, notamment: (1) en incluant d'autres groupes d'âge (des enfants plus jeunes comme des plus âgés ainsi que des adultes) ; (2) en étudiant un autre type de séquence linguistique telle la narration ou l'argumentation ; (3) enfin, nous pourrions inclure des enfants à profil atypique afin de voir si la gestualité comme le verbal peuvent être 
des indicateurs de pathologies, comme le suggère, entre autres, les travaux de Goldin-Meadow (entre autres, Goldin-Meadow, 2009 ; Ozcaliskan, Levine, et Goldin-Meadow, 2009).

\section{Références bibliographiques}

Alibali, M. W., Kita, S. et Young, A. (2000). Gesture and the process of speech production: We think, therefore we gesture. Language and Cognitive Processes, 15 (6), 593-613.

Alibali, M. W., Heath, D. C., et Myers, H. J. (2001). Effects of visibility between speaker and listener on gesture production: Some gestures are meant to be seen. Journal of Memory and Language, 44, 169-188.

Austin, J.L. (1962). How To Do Things With Words. Oxford: Clarendon Press.

Bavelas, J. B. (1994). Gesture as part of speech: Methodological implications. Research on Language and Social Interaction, 27(3), 201-221.

Bavelas, J. B., Chovil, N., Lawrie, D.A. et Wade, A.(1992). Interactive gestures. Discourse Processes, 15, 469-489.

Bavelas, J. B., Gerwing, J. Sutton, C. et Prevost D. (2007). Gesturing on the telephone: independent effects of dialogue and visibility. Journal of Memory and Language, 58, 495-520.

Beattie, G. et Shovelton, H. (2000). Iconic hand gestures and the predictability of words in context in spontaneous speech. The British Psychological Society, 91, 473-491.

Beattie, G. et Shovelton, H. (2002). An experimental investigation of some properties of individual iconic gestures that mediate their communicative power. The British Psychological Society, 93, 179-192.

Berman, R. (2008). The psycholinguistics of text construction. Journal of Child Language, 35, 1-37.

Butterworth, B. et Hadar, U. (1989). Gesture, speech, and computational stages: A reply to McNeill. Psychological Review, 96, 168-174.

Clark, H. H. (1996). Using language. Cambridge : Cambridge University Press.

Cohen, A. A. (1977). The communications functions of hand illustrators. Journal of communication, 27, 54-63.

Cohen, A. A. et Harrison, R. P. (1973). Intentionality in the use of hand illustrators in face-to-face communications situations. Journal of Personnality and Social Psychology, 28(2), 276-279.

Colletta, J.-M. (2000). A propos de la catégorisation fonctionnelle des kinèmes co-verbaux. Actes des XXIIIèmes Journées d'Etude sur la Parole, Aussois-France, 19-23 juin 2000, Grenoble: ICP, 229-232.

Colletta, J.-M. (2004). Le développement de la parole chez l'enfant âgé de 6 et 11 ans. Corps, langage et cognition. Hayen, Belgique: Mardaga.

Colletta, J.-M. (2007). Signaux corporels et acquisition du langage : des relations constantes et étroites. Langage et pratiques, 39, 20-33.

Colletta, J.-M. (2009). Comparative analysis of children's narratives at different ages: A multimodal approach. Gesture, 9, 1, 61-97.

Colletta, J.-M., Pellenq, C. (2005). Les coverbaux de l'explication chez l'enfant âgé de 3 à 11 ans. Actes du $2 e$ Congrès de l'ISGS : Interacting bodies, corps en interaction, Lyon, 15-18 juin 2005.

Colletta, J.-M. et Pellenq, C. (2009). The development of multimodal explanations in French children. In Nippold, M.A. et Scott, C.M. (éd.), Expository Discourse in Children, Adolescents, and Adults. Development and Disorders. Taylor \& Francis, 63-100.

Colletta, J.-m., Pellenq, C., Guidetti, M. (2010). Age-related changes in co-speech gesture and narrative: Evidence from French children and adults (in press). Speech Communication.

Colletta, J.-M., Kunene, R., Venouil, A., Kaufmann, V. \& Simon, J.-P. (2009). Multitrack annotation of child language and gestures. In Kipp, M., Martin, J.-C., Paggio, P. \& Heylen, D. (éd.), Multimodal Corpora. LNAI 5509. Springer.

Cosnier, J. et Brossard, A. (1984). La communication non verbale. Texte de Base en psychologie. Neuchâtel, Paris : Delachaux et Niestlé. 
Cosnier, J. (1996). Les gestes du dialogue, la communication verbale. Psychologie de la motivation, 21, 129-138.

Cosnier, J. (2004). Les gestes du dialogue. La communication, état des savoirs, 119-128.

De Ruiter, J.P. (2000). The production of gesture and speech. In McNeill, D. (éd.), Language and Gesture. Cambridge University Press, 284-311.

Doherty-Sneddon, G. et Kent, G. (1996).Visual signals and communication abilities of children. British Journal of Developmental Psychology, 18, 595-608.

Doherty-Sneddon, g., McAuley, S., Bruce, V. et Langton, S. (2000). Visual signals and children's communication: negative effects on task outcome. Bristish Journal of developpemental Psychology, 18, 595-608.

Emmorey, K. et Cassey, S. (2001). Gesture, thought and spatial language ? Gesture, 1, 35-50.

Fantazi, D. (2010). La gestualité cohésive dans les récits d'enfants âgés de 9 à 11 ans. Lidil, 42, 97-112.

Fantazi, D. et Colletta, J.-M. (2010). L'effet du contenu narratif sur la focalisation dans les gestes iconiques. Actes des XXVIIIes Journées d'étude sur la Parole, 89-92.

Goldin-Meadow, S. (2003). Hearing gesture: How our hands help us think. Cambridge, MA: Harvard University Press.

Goldin-Meadow, S. (2009). How Gesture Promotes Learning Throughout Childhood. Society for Research in Child Development, 3 (2), 106-111.

Graziano, M. (2010). Le développement des gestes pragmatiques et leur relation avec le développement de la compétence textuelle chez l'enfant âgés de 4 à 10 ans. Lidil, 42, 113-138.

Grize, J.-B. (1990). Logique et langage. Gap/Paris, Ophrys.

Hickmann, M. (2003). Children's discourse: person, space and time across languages, Cambridge ; New York: Cambridge University Press.

Jisa, H. (2004). Growing into academic French. Later Language Development : Typological and psycholinguistic Perspectives. TILAR, 3, 135-162.

Krauss, R.M., Chen, Y. et Chawla, P. (1996). Non verbal behavior and non verbal communication: What do conversational hand gestures tell us? In Zanna, M. (éd.), Advances inexperimental social psychology, 28, Tampa: AcademicPress, 389-450.

Krauss, R., Chen, Y. et Gottesman, R. F. (2000). Lexical gestures and lexical access: a process model. In McNeill, D. (éd.), Language and gesture, Cambridge, Cambridge University Press, 261-283.

Kendon, A. (1987). On gesture: its complementary relationship with speech. In Siegman, A. W. et Feldstein, S. (éd.), Nonverbal behavior ans communication, Hillsdale, NJ: Lawrence Erlbaum, 65-97.

Kendon, A. (2000). Language and Gesture: Unity or Duality. In McNeill, D. (éd.), Language and Gesture: Window into Thought and Action, Cambridge: Cambridge University Press, 47-63.

Kendon, A. (2004). Gesture: visible action as utterance. Cambridge, Cambridge University Press.

Kita, S. (2000). How representational gestures help speaking. In McNeil, D. (éd.), Language and gesture, Cambridge, Cambridge University Press, 141-161.

Kita, S. et Özyürek, A. (2003). What does cross-linguistic variationin semantic coordination of speech and gesture reveal?: Evidence for an interface representation of spatial thinking and speaking. Journal of Memory and Language, 48, 16-32.

Kita, S., Özyürek, A., Allen, S., Brown, A., Furman, R. et Ishizuka, T. (2007). Relations between syntactic encoding and co-speech gestures: Implications for a model of speech and gesture production. Language and cognitive processes, 22(8), 1212-1236

Krauss, R. M., Dushay, R. A., Chen, Y. et Rausher, F. (1995). The communicative value of conversational hand gestures. Journal of Experimental Social Psychology, 31(6), 533-552.

Levelt, W. (1989). Speaking: From intention to articulation. Cambridge/Massachussetts: The MIT Press. 
Lozano, S.C. \& Tversky, B. 2006. Communicative gestures facilitate problem solving for both communicators and recipients. Journal of Memory and Language 55, 47-63.

Lund, K. (2003). Analyse de l'activité explicative en interaction : étude de dialogues d'enseignants de physique en formation interprétant les interactions entre élèves. Thèse de doctorat, Université de Lyon.

Mazur-Palandre, A. (2009). Le flux de l'information, aspects syntaxiques et discursifs, Une étude fonctionnaliste et développementale. Thèse de doctorat, Université de Lyon.

McNeill, D. (1992). Hand and Mind: What gestures reveal about thought. Chicago, Chicago University Press.

McNeill, D. (2000). Language and gesture. Cambridge, Cambridge University Press.

McNeill, D. (2002). Gesture and Language Dialectic. Acta Linguistica Hafniesia.

McNeill, D. et Duncan, S. D. (2000). Growth Point in thinking-for-speaking. In McNeill, D. (éd.), Language and Gesture: Window into Thought and Action. Cambridge: Cambridge University Press, 141-161.

Ozcaliskan, S.,Levine, S. et Goldin-Meadow, S. (2009). Gesturing with an injured brain: How gesture helps children with early brain injury learn linguistic constructions. Manuscript submitted for publication.

Ravid, D. (2006). Semantic development in textual contexts during the school years: Noun Scale analyses, Journal of Child Language, 33, 791-821.

Ravid, D. et Berman, R. (2010). Developing noun phrase complexity across adolescence: A text-embedded analysis, First Language, 30(1), 1-29.

Rimé, B. (1982). The elimination of visible behavior from social interactions: Effects on verbal, non verbal ans interpersonal variables. European Journal of Social Psychology, 12, 113-129.

Schegloff, E.A. (1984). On some gestures relation to speech. In Atkinson, J.M. et Heritage, J. (éd.), Structures of social action: Studies in conversation alanalysis. Cambridge: Cambridge University Press.

Schneuwly, B. (1988). Le langage écrit chez l'enfant, Neuchâtel: Delachaux et Niestlé.

Traxler, M. et Gernsbacher, M. (1995). Improving coherence in written communication. In Gernsbacher, M. and Givón, T. (éd.), Coherence in spontaneous text, Philadelphia: John Benjamins, 215-238

Veneziano, E. et Hudelot, C. (2002). Développement des compétences pragmatiques et théories de l'esprit chez l'enfant : le cas de l'explication. In Bernicot, J., Trognon, A., Guidetti, M. et Musiol, M. (éd.), Pragmatique et psychologie, Nancy : Presses Universitaires de Nancy, 215-236.

Veneziano, E. et Sinclair, H. (1995). Functional changes in early child language: the appearance of references to the past and of explanations.Journal of Child Language, 22, 557-581.

\section{Remerciements}

Nous tenons à remercier : les instituteurs, parents et enfants pour leur participation de Tignieu-Jameyzieu et Saint-Romain-de-Jalionas; les deux expérimentateurs, Clément Moret et Nathalie Besacier; les statisticiennes, Céline Faure et Marilyne Goutagny et enfin Gregory Pescini (CNRS) et Jean-Marc Colletta (Laboratoire Lidilem) pour leur aide et conseils.

\footnotetext{
${ }^{1}$ Il s'agit du projet PAMEALE (Publication Adaptative Multi-Ecrans d'Activités Ludo-Educatives) qui est financé par l'Europe et la Région Rhône-Alpes (FEDER).

${ }^{2}$ Notons que déjà Cosnier et Brossard en 1984 pensait, en d'autres termes, multimodalité, impact du contexte, gestes et langage comme système intégré, synchronicité geste/langage, influence des gestes sur la parole et de la parole sur les gestes en proposant le modèle de l'activité énonciative.

${ }^{3}$ Notons que les conditions d'application de l'analyse de variance (échantillonnage au hasard, indépendance des observations, distribution normales et homogénéité des variances du facteur inter-sujets - test de Levene) sont remplies.
} 